\title{
Swedish snuff (snus) and risk of cardiovascular disease and mortality: prospective cohort study of middle-aged and older individuals
}

\author{
Olga E. Titova' ${ }^{1}$, John A. Baron ${ }^{1,2,3}$, Karl Michaëlsson ${ }^{1}$ and Susanna C. Larsson ${ }^{1,4^{*}}$ (D)
}

\begin{abstract}
Background: Cigarette smoking is a well-known risk factor for cardiovascular disease (CVD), but whether smokeless tobacco such as snuff is associated with the risk of CVD is still unclear. We investigated the association of the use of Swedish oral moist snuff (snus) with a broad range of CVDs and CVD mortality.

Methods: We used data from a population-based cohort of 41,162 Swedish adults with a mean baseline age of 70 (56-94) years who completed questionnaires regarding snus use and other lifestyle habits and health characteristics. Participants were followed up for incident cardiovascular outcomes and death over 8 years through linkage to the Swedish National Patient and Death Registers. Hazard ratios (HR) were estimated by Cox proportional hazards regression. We conducted analyses among all subjects as well as among never smokers to reduce residual confounding from smoking.

Results: After adjustment for smoking and other confounders, snus use was not associated with myocardial infarction, heart failure, atrial fibrillation, aortic valve stenosis, abdominal aortic aneurysm, stroke, or CVD mortality. However, in never smokers, snus use was associated with a statistically significant increased risk of total and ischemic stroke (HRs [95\% confidence intervals] $=1.52[1.01-2.30]$ and $1.63[1.05-2.54]$, respectively) and non-significantly positively associated with some other CVDs.

Conclusions: In this middle-aged and elderly Swedish population, current Swedish snus use was not associated with the risk of major heart and valvular diseases, abdominal aortic aneurysm, or CVD mortality in the entire study population, but was linked to an increased risk of stroke in never smokers.
\end{abstract}

Keywords: Cardiovascular disease, Snus, Snuff, Smokeless tobacco, Mortality

\section{Background}

Cigarette smoking is one of the leading causes of morbidity and premature death worldwide [1]. The adverse effect of cigarette smoking on cardiovascular health has been well documented in prospective cohort studies [1-

\footnotetext{
* Correspondence: susannalarsson@surgsciuu.se

'Department of Surgical Sciences, Unit of Medical Epidemiology, The EpiHub, Uppsala University, Dag Hammarskjölds väg 14 B, 75185 Uppsala, Sweden

${ }^{4}$ Unit of Cardiovascular and Nutritional Epidemiology, Institute of Environmental Medicine, Karolinska Institutet, Stockholm, Sweden Full list of author information is available at the end of the article
}

7]. A recent Mendelian randomization study provided further evidence for causal association between smoking and a broad range of cardiovascular diseases (CVDs), including coronary artery disease, heart failure (HF), abdominal aortic aneurysm (AAA), and ischemic stroke [8]. Consequently, strategies to reduce cigarette smoking are of great importance globally. There is considerable discussion regarding the effectiveness of nicotine replacement therapy, and safety of the alternative nicotine delivery products such as smokeless tobacco, especially

(c) The Author(s). 2021 Open Access This article is licensed under a Creative Commons Attribution 4.0 International License, which permits use, sharing, adaptation, distribution and reproduction in any medium or format, as long as you give appropriate credit to the original author(s) and the source, provide a link to the Creative Commons licence, and indicate if changes were made. The images or other third party material in this article are included in the article's Creative Commons licence, unless indicated otherwise in a credit line to the material. If material is not included in the article's Creative Commons licence and your intended use is not permitted by statutory regulation or exceeds the permitted use, you will need to obtain permission directly from the copyright holder. To view a copy of this licence, visit http://creativecommons.org/licenses/by/4.0/. The Creative Commons Public Domain Dedication waiver (http://creativecommons.org/publicdomain/zero/1.0/) applies to the data made available in this article, unless otherwise stated in a credit line to the data. 
among those who are less likely or unwilling to quit smoking $[9,10]$.

Smokeless tobacco products may vary considerably in composition and the amount of potentially toxic compounds [11]. Oral moist snuff (snus) is commonly used in Sweden and some other Scandinavian countries as well as in the USA. Swedish snus is used loose or in a portion-packed form (sachets) and is typically placed between the lip and the gum [11]. Compared to the late 1980 s, there was an increase in snus consumption in Sweden, accompanied by a considerable decline in prevalence of cigarette smoking [12]. During 2016-2017, $22 \%$ of Swedish men and 5\% of women were daily snus users and $11 \%$ of the population were daily smokers according to the central statistical agency in Sweden [12]. Despite the absence of harmful combustion products, the amount of nicotine in smokeless tobacco is comparable to that in cigarettes and the peak blood concentration of nicotine in users is similar to those observed in cigarette smokers $[13,14]$. Nicotine raises heart rate and blood pressure regardless of the route of administration [15-17]. However, the long-term effect of moist snuff on the cardiovascular system has not been thoroughly studied and results remain inconsistent.

Previous research regarding the association between snuff use and CVD has mainly focused on CVD-specific mortality, incidence of ischemic heart disease, myocardial infarction (MI), and stroke [18-20]. An increased risk of CVD or CVD-related death was demonstrated in some [21, 22], but not all, prospective studies [23-25]. Studies of the association between snuff use and other CVDs, such as heart failure (HF) [21], atrial fibrillation (AF) [25], aortic valve stenosis (AVS), and AAA are scarce or absent.

The aim of this study was to investigate the associations of Swedish snus use with specific CVD events, including MI, HF, AF, AVS, AAA, and stroke, as well as CVD mortality in a cohort of 41,162 Swedish men and women (56-94 years of age). Cigarette smoking has previously been shown to be significantly associated with each of these CVDs [2-7] except AF [26] in this study population, but the association between snus use and CVD was not investigated.

\section{Methods}

\section{Study population}

The data from the National Research Infrastructure SIMP LER (Swedish Infrastructure for Medical Population-based Life-course Environmental Research) was used in this analysis. A detailed description can be found elsewhere (https://www.simpler4health.se). Information on lifestyle and other risk factors for CVD and other diseases was obtained with structured questionnaires in 2008/2009. In the present analysis, we excluded individuals who died or had a study endpoint prior to July 1, 2009, and those who had missing information on snus use or cigarette smoking (Additional file 1). This left 41,162 eligible participants (18,027 women and 23,135 men) with a mean baseline age of 70 (56-94) years. In the main analysis of each CVD outcome, we excluded participants with a diagnosis of the corresponding specific CVD before start of a follow-up (e.g., those with MI before baseline were excluded from the analysis of MI; those who were diagnosed with any stroke before baseline, were excluded from the analyses of stroke and stroke types), as ascertained through linkage to the Swedish National Patient Register from 1987 and based on the International Classification of Diseases (ICD)-9 and ICD-10 codes. In the analysis of overall CVD mortality, those who were diagnosed with any CVD before the start of a follow-up were excluded. The number of prevalent CVD cases excluded in each analysis is shown in Additional file 1.

\section{Assessment of snus use, potential confounders, and intermediates}

In $2008 / 2009$, participants completed a Health questionnaire and Diet and lifestyle questionnaire that included information about snus use, smoking status, alcohol consumption, educational attainment, weight, height, physical activity, and history of diabetes, hypertension, and hypercholesterolemia. Participants were asked if they used snus regularly (more than 5 portions of snus/week) with the following options: no; yes, currently; yes, in the past. In addition, former snus users $(n=2946,7 \%$ of the entire cohort) indicated how many years ago they stopped using snus. As most ever using participants stopped using snus long before the start of the follow-up (median; quartile 1-quartile 3; 20 (7-28) years), former snus users and non-users were combined into one group for analysis. Participants were also asked if they smoked cigarettes regularly (more than 5 cigarettes/week) with the following options: no; yes, currently; yes, in the past. Participants who answered that they never smoked cigarettes regularly were defined as never smokers. A participant was considered to have a history of diabetes if he/ she reported having diabetes and/or diabetes treatment.

\section{Case ascertainment and follow-up}

Incident cases of CVDs that occurred after the start of follow-up were ascertained through linkage with the Swedish National Patient Register (covering both in- and out-patients) and the Cause of Death Register using the unique personal identity number assigned to each Swedish resident and classified according to the ICD 10th Revision codes. The endpoints in the present study were acute MI (I21), HF (I50 and I11.0), AF (I48), AVS (I35.0 and I35.2), AAA (I71.3 and I71.4), ischemic stroke (I63), intracerebral hemorrhage (I61), subarachnoid hemorrhage (I60), 
unspecified stroke (I62), and CVD mortality (I00-I99) as the primary cause of death. Participants were followed up from July 1, 2009, to the first date of diagnosis of specific CVD or CVD-mortality, death from any cause, or December 31, 2017, whichever occurred first.

\section{Statistical analysis}

Descriptive data are presented as mean (standard deviation) for continuous variables and as the number of participants (\%) for categorical variables. Numerical data were analyzed with the Mann-Whitney $U$ test. The Pearson chi-square test was used to analyze group differences for categorical variables. Cox proportional hazards regression models were used to obtain hazard ratios (HR) with 95\% confidence intervals (CI) with age as the time scale and adjusted for sex (as a stratification variable) in the basic model. Snus use status was classified as non-use (never used snus regularly or former users) and current snus use at baseline.

First, we investigated the associations of snus use and CVD risk in the population-based cohort, including all participants in the analysis. In a first multivariable model, we further adjusted for education (less than high school, high school, or university), smoking status (never, former, current smokers), walking/bicycling (never/seldom; $<20$ $\mathrm{min} /$ day; $20-40 \mathrm{~min} /$ day; $>40 \mathrm{~min} /$ day), alcohol intake (never drinkers; past or current drinkers of $<1$ drink/ week; $1-<7$ drinks/week; 7-<15 drinks/week; 15-21 drinks/week; > 21 drinks/week), and exercise (almost never; < $1 \mathrm{~h} /$ week; $1 \mathrm{~h} /$ week; $2-3 \mathrm{~h} /$ week; 4-5 h/week; $\geq 5$ $\mathrm{h} /$ week). In a second multivariable model, we further adjusted for potential intermediates of the association of snus use with CVD risk, including body mass index (weight divided by the square of height; <22.5, 22.5-24.9, $25.0-29.9$, or $\geq 30 \mathrm{~kg} / \mathrm{m}^{2}$ ) and history of diabetes (no/yes), hypertension (no/yes), and hypercholesterolemia (no/yes). For categorical variables, the category with the lowest value was treated as the reference group, except for body mass index where normal weight $\left(22.5-24.9 \mathrm{~kg} / \mathrm{m}^{2}\right)$ was used as the reference.

The proportion of missing data on the potential confounders/intermediates used in the main analysis was less than $5 \%$. A separate category ("missing") was created for each variable containing missing values. To reduce confounding from cigarette smoking, we conducted a separate analysis of the association between snus use and CVD risk among individuals who reported never smoking regularly. Proportional hazard assumptions were assessed by Schoenfeld's test. Potential confounders were selected using directed acyclic graphs (DAGs) [27] based on our a priori knowledge of the relationships among potential confounders, intermediate variables, exposure, and outcome variables, as well as on existing information regarding factors associated with CVD and tobacco consumption [28, 29]. With regard to HF, we performed a sensitivity analysis additionally excluding individuals with a diagnosis of MI before the start of a follow-up. In addition, we conducted analyses using never snus users as the reference group; as well as analyses including only men. All statistical tests were two sided. All statistical analyses were performed using Stata version 15.1 (StataCorp, College Station, TX, USA).

\section{Results}

Baseline characteristics of study participants according to snus use are shown in Table 1. Compared with nonusers, current snus users were younger, had lower educational attainment, were more likely to be men, had higher alcohol intake, were more likely to be former or current cigarette smokers, were less physically active, had higher BMI, and were less likely to report hypertension at the baseline $(p<0.05)$. As the majority of snus users were men, we compared these baseline characteristics for men non-users and men snus users. The results were similar as for the entire cohort, except for history of hypertension which did not differ between non-users and snus users (data not shown).

The number of incident CVDs and CVD-related deaths during up to 8 years of follow-up is shown in Table 2. In age- and sex-adjusted analysis, current snus use was associated with increased risk of AF and AAA compared with non-use, but these associations did not remain after adjustment for cigarette smoking (the major confounder) and other risk factors (Table 2). There was no association between snus use and the other CVDs.

After restriction of the database to never-smokers, current snus use was associated with a significant increased risk of total stroke and ischemic stroke (multivariable $\mathrm{HRs}[95 \% \mathrm{CIs}]=1.52[1.01-2.30]$ and 1.63 [1.05-2.54], respectively) (Table 3, Fig. 1). In age- and sex-adjusted analysis, current snus use was associated with an increased risk of CVD death, but this association did not remain statistically significant after adjustment for potential confounders. In addition, a trend for an increased risk of MI and AF in snus users was observed, but results did not attain statistical significance (Table 3, Fig. 1). The results for AVS, AAA, and hemorrhagic stroke were not shown due to the small number of cases in the group of snus users $(n<5)$.

In a sensitivity analysis, the results for HF were similar after additional exclusion of individuals with MI before baseline (HR 1.05, 95\% CI 0.84-1.32, multivariable model 2 in the entire sample; HR $0.81,95 \%$ CI $0.44-$ 1.49, multivariable model 2 in never smokers). The results for current snus users were similar for all CVD outcomes when using "never snus users" as a reference group (data not shown). In addition, we performed 
Table 1 Baseline characteristics according to snus use in 41,162 Swedish middle-aged and elderly participants, 2009-2017

\begin{tabular}{|c|c|c|}
\hline \multirow[t]{2}{*}{ Characteristics* } & \multicolumn{2}{|l|}{ Snus use } \\
\hline & Nonusers & Users \\
\hline Number of participants, $n$ (\% of total) & $38,862(94.4)$ & $2300(5.6)$ \\
\hline Age at baseline, years, mean (SD) & $69.8(8.0)$ & $65.5(6.7)$ \\
\hline Men, $n(\%)$ & $20,988(54.0)$ & $2147(93.4)$ \\
\hline \multicolumn{3}{|l|}{ Education, $n(\%)$} \\
\hline$\leq 9$ years & $11,733(30.2)$ & $716(31.1)$ \\
\hline $10-12$ years & $18,104(46.6)$ & $1164(50.6)$ \\
\hline$>12$ years & $89,291(23.0)$ & $412(17.9)$ \\
\hline Unknown & $96(0.25)$ & $8(0.35)$ \\
\hline \multicolumn{3}{|l|}{ Cigarette smoking status, $n(\%)$} \\
\hline Non-smokers & $21,099(54.3)$ & $418(18.2)$ \\
\hline Former smokers & $14,411(37.1)$ & $1590(69.1)$ \\
\hline Current smokers & $3352(8.6)$ & $292(12.7)$ \\
\hline \multicolumn{3}{|l|}{ Alcohol intake, $n(\%)$} \\
\hline Never drinkers & $3689(9.5)$ & $18(0.8)$ \\
\hline Past or current drinkers of $<1$ drink/week & $8198(21.1)$ & $328(14.3)$ \\
\hline $1-<7$ drinks/week & $18,605(47.9)$ & $1148(49.9)$ \\
\hline $7-<15$ drinks/week & $7085(18.2)$ & $644(28.0)$ \\
\hline 15-21 drinks/week & $957(2.5)$ & $116(5.0)$ \\
\hline$>21$ drinks/week & $328(0.8)$ & $46(2.0)$ \\
\hline \multicolumn{3}{|l|}{ Walking/bicycling, $n(\%)$} \\
\hline Never/seldom & $2401(6.2)$ & $173(7.5)$ \\
\hline$<20 \mathrm{~min} /$ day & $6652(17.1)$ & $514(22.4)$ \\
\hline 20-40 min/day & $16,215(41.7)$ & $869(37.8)$ \\
\hline$>40 \mathrm{~min} /$ day & $13,031(33.5)$ & $719(31.3)$ \\
\hline Unknown & $563(1.5)$ & $25(1.1)$ \\
\hline \multicolumn{3}{|l|}{ Exercise, $n(\%)$} \\
\hline Almost never & $20,868(53.7)$ & $1515(65.9)$ \\
\hline$<1 \mathrm{~h} /$ week & $4694(12.1)$ & $256(11.1)$ \\
\hline $1 \mathrm{~h}$ & $6623(17.0)$ & $234(10.2)$ \\
\hline $2-3 h$ & $5020(12.9)$ & $211(9.2)$ \\
\hline $4-5 \mathrm{~h}$ & $705(1.8)$ & $44(1.9)$ \\
\hline$\geq 5 \mathrm{~h} /$ week & $268(0.7)$ & $8(0.4)$ \\
\hline Unknown & $684(1.8)$ & $32(1.4)$ \\
\hline \multicolumn{3}{|l|}{ Body mass index category, $\mathrm{kg} / \mathrm{m}^{2}, n(\%)$} \\
\hline $22.5-24.9$ & $10,035(25.8)$ & $523(22.7)$ \\
\hline$<22.5$ & $6553(16.9)$ & $227(9.9)$ \\
\hline $25.0-29.9$ & $16,053(41.3)$ & $1109(48.2)$ \\
\hline$\geq 30.0$ & $4990(12.8)$ & $375(16.3)$ \\
\hline Unknown & $1231(3.2)$ & $66(2.9)$ \\
\hline Hypertension, $n(\%)$ & $16,214(41.7)$ & $887(38.6)$ \\
\hline Hypercholesterolemia, $n(\%)$ & $9832(25.3)$ & $589(25.6)$ \\
\hline \multicolumn{3}{|l|}{ Diabetes, $n(\%)$} \\
\hline No & $34,188(88.0)$ & $2005(87.2)$ \\
\hline Yes & $3560(9.2)$ & $225(9.8)$ \\
\hline Unknown & $1114(2.9)$ & $70(3.0)$ \\
\hline
\end{tabular}


analyses including only men and found similar results to those presented in Tables 2 and 3 (data not shown).

\section{Discussion}

In this study, we investigated the association between snus use and the subsequent risk of several CVDs and CVD mortality in a cohort of middle-aged and older men and women. Findings from the analyses adjusted for smoking that are based on the entire cohort, do not support a detrimental effect of snus use on the risk of major heart and valvular diseases, AAA, or CVD mortality. However, in never smokers, snus use was associated with an increased risk of stroke, particularly ischemic stroke, and nonsignificantly associated with increased risk of myocardial infarction, atrial fibrillation, and CVD death.

\section{Comparisons with other studies}

Several health hazards associated with smokeless tobacco use have previously been documented, including oral cancer [30], CVD [31], and all-cause and causespecific mortality [32]. Our findings of no significant association between snus use and incidence of $\mathrm{MI}$ and $\mathrm{AF}$ are in agreement with previous reports [18, 25, 33, 34]. For example, in a pooled analysis of eight Swedish prospective cohort studies, current snus use was not related to risk of acute MI [18]. However, long-term use of snus was associated with an increased relative risk of fatal MI, especially among heavy users, in a cohort of nonsmoking male construction workers (mean baseline age of 31.5 years and 19 years of follow-up) [33]. Similar to our results, snus use was not associated with AF risk in a pooled analysis of 7 Swedish prospective cohorts of never-smoking men [25].

In contrast to our finding, a previous Swedish study demonstrated that snus use was associated with a higher risk of HF in elderly men after adjusting for smoking status $(n=1076 ; \mathrm{HR}, 2.08 ; 95 \% \mathrm{CI}, 1.03-4.22)$ and in younger never-smoking male construction workers $(n=$ 118,425; HR, 1.28; 95\% CI, 1.00-1.64) [21]. Baseline information in this previous study was collected in 19911995 (cohort of elderly men) vs 2008-2009 in our study and might be related to differences in lifestyle and snus composition during these time periods. For example, a higher proportion of current cigarette smokers was observed among elderly men snus users [21] compared to our study. Moreover, the amount of some potentially toxic compounds such as tobacco-specific nitrosamines and some polycyclic aromatic hydrocarbons (PAH) was substantially reduced in Swedish snus in the early 2000s; and the GothiaTek standard that regulates the content of such undesired components and specifies manufacturing standards, was introduced in 2000 [11]. In addition, in the analysis of elderly men [21], the association of snus use with HF risk among never cigarette smokers was not examined, likely resulting in residual confounding from smoking. Another study based on two US prospective cohorts reported higher CVD mortality among men who currently used chewing tobacco or snuff compared with non-users [22]. In the present study, an increased risk of CVD death among snus users with no history of cigarette smoking was observed after adjustment for age and sex only, but the association did not persist after adjustment for other confounders.

We are not aware of any previous study on snuff use in relation to risk of AVS or AAA. In our study population, cigarette smoking was previously shown to be significantly associated with an increased risk of these outcomes $[3,7]$, with a particularly strong association with AAA (almost 7-fold and 11-fold increased risks in heavy smoking men and women, respectively) [7].

Our findings of a positive association between current snus use and risk of total stroke and ischemic stroke in never smokers indicate that nicotine per se may contribute to the pathophysiology of stroke. This is in line with previous reports of an increased risk of stroke fatality among snuff or chewing tobacco users compared to non-users [22, 23, 35]. However, a recent meta-analysis of studies conducted in the USA and Sweden, demonstrated an increased risk of stroke among USA but not in Swedish smokeless tobacco users [19]. Thus, several Swedish studies have found no association between the use of snus and incident stroke in non-smokers or in the entire sample analysis adjusted for smoking status [23, $24,34,36]$. Importantly, these prospective cohort studies included younger participants compared to the present study. For example, in the pooled analysis of eight Swedish prospective cohort studies, including never-smoking men, no association between the use of snus and the risk of overall stroke or any stroke types were observed [23]. The baseline mean age in this pooled study was 35 years vs 70 years in our study. The possible explanation that we see the effect of snus use on the risk of stroke only in never smokers is that the majority of snus users in our study are former smokers (69\%). Cigarette smoking is a well-established strong risk factor for CVDs and may mask the effect of snus in the analysis based on the entire cohort, i.e. residual confounding takes place. In addition, survival bias could occur especially in such cohort of middle-aged and elderly participants, i.e., some former and current smokers could die before the baseline or be excluded from the analysis due to a cardiovascular event prior baseline.

As in cigarettes, nicotine is the main alkaloid in snuff. A number of animal and human studies indicated that nicotine may contribute to CVD via multiple pathways [37-40]. For example, hypertension is the main risk factor for stroke. While epidemiological findings on snuff use and hypertension are inconclusive [13, 16], 
Table 2 Hazard ratios (95\% confidence intervals) of CVDs according to snus use in the entire study population of Swedish adults, 2009-2017

\begin{tabular}{|c|c|c|}
\hline \multirow[b]{2}{*}{ Outcome and model (total number) } & \multicolumn{2}{|l|}{ Snus use } \\
\hline & Nonusers & Users \\
\hline \multicolumn{3}{|l|}{ Myocardial infarction $(N=38,844)$} \\
\hline Total number of cases & 1610 & 97 \\
\hline Age and sex-adjusted model & 1.00 (reference) & $1.01(0.82-1.25)$ \\
\hline Multivariable model $1^{\dagger}$ & 1.00 (reference) & $0.95(0.77-1.18)$ \\
\hline Multivariable model $2^{\dagger+}$ & 1.00 (reference) & $0.96(0.78-1.19)$ \\
\hline \multicolumn{3}{|l|}{ Heart failure $(N=40,326)$} \\
\hline Total number of cases & 1990 & 96 \\
\hline Age and sex-adjusted model & 1.00 (reference) & $1.15(0.94-1.42)$ \\
\hline Multivariable model $1^{\dagger}$ & 1.00 (reference) & $0.99(0.80-1.23)$ \\
\hline Multivariable model $2^{\text {t† }}$ & 1.00 (reference) & $1.00(0.81-1.23)$ \\
\hline \multicolumn{3}{|l|}{ Atrial fibrillation $(N=38,044)$} \\
\hline Total number of cases & 3991 & 234 \\
\hline Age and sex-adjusted model & 1.00 (reference) & $1.18(1.03-1.35)$ \\
\hline Multivariable model $1^{\dagger}$ & 1.00 (reference) & $1.11(0.97-1.27)$ \\
\hline Multivariable model $2^{\dagger \dagger}$ & 1.00 (reference) & $1.11(0.97-1.28)$ \\
\hline \multicolumn{3}{|l|}{ Aortic valve stenosis $(N=40,873)$} \\
\hline Total number of cases & 421 & 19 \\
\hline Age and sex-adjusted model & 1.00 (reference) & $0.86(0.54-1.37)$ \\
\hline Multivariable model $1^{\dagger}$ & 1.00 (reference) & $0.82(0.51-1.32)$ \\
\hline Multivariable model $2^{\dagger+}$ & 1.00 (reference) & $0.83(0.52-1.33)$ \\
\hline \multicolumn{3}{|l|}{ Abdominal aortic aneurysm $(N=40,853)$} \\
\hline Total number of cases & 480 & 55 \\
\hline Age and sex-adjusted model & 1.00 (reference) & $1.37(1.03-1.81)$ \\
\hline Multivariable model $1^{\dagger}$ & 1.00 (reference) & $1.06(0.79-1.40)$ \\
\hline Multivariable model $2^{\dagger+}$ & 1.00 (reference) & $1.07(0.80-1.42)$ \\
\hline \multicolumn{3}{|l|}{ Total stroke $^{*}(N=39,399)$} \\
\hline Total number of cases & 2071 & 105 \\
\hline Age and sex-adjusted model & 1.00 (reference) & $1.09(0.89-1.34)$ \\
\hline Multivariable model $1^{\dagger}$ & 1.00 (reference) & $1.04(0.85-1.27)$ \\
\hline Multivariable model $2^{\text {t† }}$ & 1.00 (reference) & $1.04(0.85-1.27)$ \\
\hline \multicolumn{3}{|l|}{ Total ischemic stroke $(N=39,399)$} \\
\hline Total number of cases & 1726 & 88 \\
\hline Age and sex-adjusted model & 1.00 (reference) & $1.12(0.90-1.39)$ \\
\hline Multivariable model $1^{\dagger}$ & 1.00 (reference) & $1.05(0.84-1.31)$ \\
\hline Multivariable model $2^{\dagger \dagger}$ & 1.00 (reference) & $1.05(0.84-1.32)$ \\
\hline \multicolumn{3}{|l|}{ Total hemorrhagic stroke $(N=39,399)$} \\
\hline Total number of cases & 317 & 20 \\
\hline Age and sex-adjusted model & 1.00 (reference) & $1.21(0.76-1.93)$ \\
\hline Multivariable model $1^{\dagger}$ & 1.00 (reference) & $1.16(0.73-1.86)$ \\
\hline Multivariable model $2^{\text {t† }}$ & 1.00 (reference) & $1.15(0.72-1.85)$ \\
\hline
\end{tabular}

CVD mortality $(N=34,355)$ 
Table 2 Hazard ratios (95\% confidence intervals) of CVDs according to snus use in the entire study population of Swedish adults, 2009-2017 (Continued)

\begin{tabular}{|c|c|c|}
\hline \multirow[b]{2}{*}{ Outcome and model (total number) } & \multicolumn{2}{|l|}{ Snus use } \\
\hline & Nonusers & Users \\
\hline Age and sex-adjusted model & 1.00 (reference) & $1.17(0.89-1.53)$ \\
\hline Multivariable model $1^{+}$ & 1.00 (reference) & $1.03(0.78-1.35)$ \\
\hline Multivariable model $2^{+\dagger}$ & 1.00 (reference) & $1.02(0.78-1.35)$ \\
\hline
\end{tabular}

Cl confidence interval, $H R$ hazard ratio. Participants with a diagnosis of the corresponding CVD before the start of a follow-up were excluded from the diseasespecific analysis. In the analysis of overall CVD mortality, those who were diagnosed with CVD before the start of a follow-up were also excluded *Includes ischemic stroke, intracerebral hemorrhage, subarachnoid hemorrhage, and undefined type of stroke

${ }^{\dagger}$ The Cox proportional hazards regression model was adjusted for age (underlying time scale), sex (as a stratification variable), education, cigarette smoking, alcohol consumption, walking/bicycling, and exercise

${ }^{+\dagger}$ The Cox proportional hazards regression model was further adjusted for potential intermediates: body mass index (categorical), and history of hypertension, hypercholesterolemia, and diabetes

Table 3 Hazard ratios (95\% confidence intervals) of CVDs according to snus use in Swedish adults who reported never smoking cigarettes regularly, 2009-2017

\begin{tabular}{|c|c|c|}
\hline \multirow[b]{2}{*}{ Outcome and model (total number) } & \multicolumn{2}{|l|}{ Snus use } \\
\hline & Nonusers & Users \\
\hline \multicolumn{3}{|l|}{ Myocardial infarction $(N=20,501)$} \\
\hline Total number of cases & 852 & 21 \\
\hline Age and sex-adjusted model & 1.00 (reference) & $1.35(0.87-2.10)$ \\
\hline Multivariable model $1^{\dagger}$ & 1.00 (reference) & $1.36(0.87-2.11)$ \\
\hline \multicolumn{3}{|l|}{ Heart failure $(N=21,130)$} \\
\hline Total number of cases & 1066 & 14 \\
\hline Age and sex-adjusted model & 1.00 (reference) & $1.04(0.61-1.78)$ \\
\hline Multivariable model $1^{\dagger}$ & 1.00 (reference) & $0.92(0.54-1.57)$ \\
\hline \multicolumn{3}{|l|}{ Atrial fibrillation $(N=19,852)$} \\
\hline Total number of cases & 2193 & 43 \\
\hline Age and sex-adjusted model & 1.00 (reference) & $1.33(0.98-1.81)$ \\
\hline Multivariable model $1^{\dagger}$ & 1.00 (reference) & $1.29(0.95-1.75)$ \\
\hline \multicolumn{3}{|l|}{ Total stroke $(N=20,620)$} \\
\hline Total number of cases & 1160 & 24 \\
\hline Age and sex-adjusted model & 1.00 (reference) & $1.57(1.04-2.37)$ \\
\hline Multivariable model $1^{\dagger}$ & 1.00 (reference) & $1.53(1.02-2.32)$ \\
\hline \multicolumn{3}{|l|}{ Total ischemic stroke $(N=20,620)$} \\
\hline Total number of cases & 957 & 21 \\
\hline Age and sex-adjusted model & 1.00 (reference) & $1.69(1.09-2.63)$ \\
\hline Multivariable model $1^{\dagger}$ & 1.00 (reference) & $1.65(1.06-2.57)$ \\
\hline \multicolumn{3}{|l|}{ CVD mortality $(N=18,189)$} \\
\hline Total number of deaths & 780 & 15 \\
\hline Age and sex-adjusted model & 1.00 (reference) & $1.70(1.01-2.87)$ \\
\hline Multivariable model $1^{+}$ & 1.00 (reference) & $1.58(0.94-2.67)$ \\
\hline
\end{tabular}

Cl confidence interval, $H R$ hazard ratio. Participants with a diagnosis of the corresponding CVD before the start of a follow-up, were excluded from the diseasespecific analysis. In the analysis of overall CVD mortality, those who were diagnosed with CVD before the start of a follow-up were also excluded *Includes ischemic stroke, intracerebral hemorrhage, subarachnoid hemorrhage, and undefined type of stroke

${ }^{\dagger}$ The Cox proportional hazards regression model was adjusted for age (underlying time scale), sex (as a stratification variable), education, alcohol consumption, walking/bicycling, and exercise 


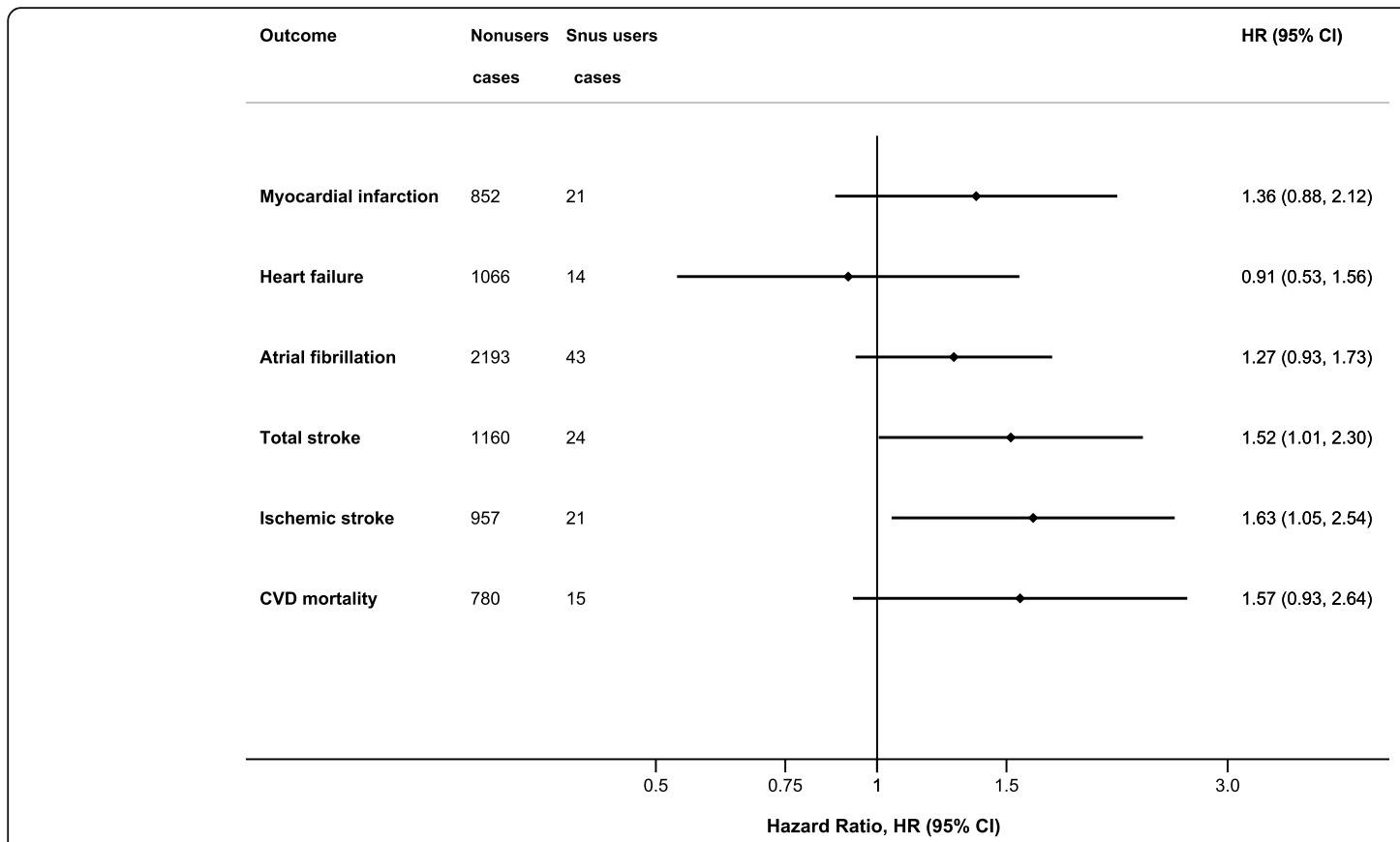

Fig. 1 Multivariable HRs ( $95 \% \mathrm{Cl}$ ) of cardiovascular diseases (CVD) and CVD death according to snus use in participants who reported never smoking cigarettes regularly. The Cox proportional hazards regression models were adjusted for age (underlying time scale), sex (as a stratification variable), education, alcohol consumption, walking/bicycling, exercise, body mass index, and history of hypertension, hypercholesterolemia, and diabetes. Cl, confidence interval; $H R$, hazard ratio

experimental evidence suggests that nicotine per se (e.g., nicotine infusion) and smokeless tobacco use may acutely increase blood pressure and heart rate as well as cause endothelial dysfunction in healthy volunteers [3841]. These adverse effects of nicotine might be related to nicotine-induced imbalance in the homeostasis of the renin-angiotensin system, an important regulatory peptide hormone component of the cardiovascular system [42]. In addition, nicotine may play a role in the enhancement of arterial wave reflection to the aorta, an indirect measure of arterial stiffness and an important determinant of central blood pressure [43]. Nicotine may also induce cardiac arrhythmias [44], which increase the risk of ischemic stroke, specifically the cardioembolic stroke subtype. Unfortunately, we did not have information on ischemic stroke subtypes and thus could not assess whether the association between snus use and stroke risk was confined to cardioembolic stroke. In vivo studies have also demonstrated that chronic nicotine exposure may have an adverse effect on cerebral blood flow and blood-brain barrier and enhances the degree of brain damage following an ischemic insult $[45,46]$.

Although the amount of nicotine in smokeless tobacco (e.g., snuff, chewing tobacco) is similar to that in cigarettes, the absorption is slower in smokeless tobacco [13]. In addition, unlike cigarette smoking, snuff does not consist of harmful combustion products, and some tobacco components can be better absorbed through the airways than through the oral mucosa [47]. This may at least partially explain less adverse effects of snuff on the circulatory system compared to cigarette smoking. The effect of other snuff constituents on the cardiovascular system is unknown and is likely minor [13].

\section{Strength and limitations}

Important strengths of our study are large sample size; a broad range of CVD outcomes, objectively assessed through linkage to nationwide population-based registers; complete case identification and no loss to followup; and the ability to adjust for important confounders. In the analyses, adjusting for smoking status, we cannot rule out residual confounding by smoking and this model rather evaluates the impact of snus use on a population level. To reduce residual confounding by smoking, we performed analyses of the association between snus using and CVD risk in never smokers.

Several limitations, however, apply to the present study. We cannot rule out that survival bias may have affected our results. For example, in the entire group, smokers may have died from other causes or had a cardiovascular event before baseline and therefore were not included in the present analyses. Another limitation is that the proportion of snus users was relatively small. Duration of snus use (and of smoking before baseline) was not available and some current snus users might have quit or changed the amount of snus consumed 
during the 8-year long follow-up period. In addition, in current smokers, we were unable to account for the number of cigarettes smoked per day. The incidence of AAA, AVS, and hemorrhagic stroke in current snus users who reported never smoking cigarettes regularly was low, and therefore, results for these outcomes were not presented. Similarly, the small number of fatal CVD cases prevented us from investigating the relationship of snus use and case fatality for specific CVDs (e.g., fatal MI or stroke). In addition, the proportion of women snus users was small. In the analyses confined to never smokers, we could not rule out a modestly higher risk of MI, AF, and CVD death in snus users. Also, our results might be not generalizable to other populations due to differences in smokeless tobacco composition and form (e.g., chewing tobacco). Finally, in view of the observational nature of this study, we cannot rule out residual and unmeasured confounding.

Further large-scale health studies of snuff use are needed. Such research concerning snuff use in relation to the development of CVDs should address sex differences, dose and recency of use, and age effects of snuff use.

\section{Conclusions}

Results from this prospective cohort study of middleaged and older Swedish adults indicate that snus use is not associated with the risk of major heart and valvular diseases, AAA, or CVD mortality in the entire study population, but is linked to an increased risk of stroke in never smokers. A potential harmful effect of snus use on other CVDs in never smokers cannot be ruled out and needs further study. The results of this study are mainly applicable to the Swedish type of snuff (snus).

\section{Abbreviations}

AAA: Abdominal aortic aneurysm; AF: Atrial fibrillation; AVS: Aortic valve stenosis; BMI : Body mass index; Cl : Confidence interval; CVD: Cardiovascular diseases; HF: Heart failure; HR: Hazard ratios; MI: Myocardial infarction

\section{Supplementary Information}

The online version contains supplementary material available at https://doi. org/10.1186/s12916-021-01979-6.

Additional file 1. Flow-chart.

\section{Acknowledgements}

We would like to thank the national research infrastructure SIMPLER for provisioning of facilities and experimental support. The computations were performed on resources provided by the Swedish National Infrastructure for Computing's (www.snic.se) support for sensitive data SNIC-SENS through the Uppsala Multidisciplinary Center for Advanced Computational Science (UPPMAX) under Project SIMP2019004

\section{Authors' contributions}

O.E.T. and S.C.L. contributed to the conception and design of the study; O.E.T. acquired the data, performed the statistical analysis, and drafted the manuscript. All authors contributed to the interpretation of the results and critical revision of the manuscript for important intellectual content and approved the final version of the manuscript.

\section{Funding}

Work of the authors is supported by grants from the Geriatric Foundation, research for healthy aging, and Börjeson, Emil and Ragna Foundation (to O.E.T.), the Swedish Research Council for Health, Working Life and Welfare (Forte; grant number 2018-00123) (to S.C.L.), the Swedish Research Council (Vetenskapsrådet; grant number 2016-01042 and 2019-00977) (to S.C.L.), and the Swedish Heart-Lung Foundation (Hjärt-Lungfonden; grant number 20190247) (to S.C.L.). The study was also supported by additional grants from the Swedish Research Council (https://www.vr.se; grant no 2015-03257, 2017 00644 and 2017-06100 to KM). SIMPLER receives funding through the Swedish Research Council under the grant no 2017-00644 (to Uppsala University and KM). SNIC is financially supported by the Swedish Research Council. The funding sources had no role in the design and conduct of the study; collection, management, analysis, and interpretation of the data; preparation, review, or approval of the manuscript; and decision to submit the manuscript for publication. Open Access funding provided by Uppsala University.

\section{Availability of data and materials}

The data that support the findings of this prospective cohort study are available upon application to the Swedish Infrastructure for Medical Population-based Life-course Environmental Research (SIMPLER; https://www. simpler4health.se).

\section{Declarations}

\section{Ethics approval and consent to participate}

The study was conducted following the Helsinki declaration, and the current data analysis was approved by the Swedish Ethical Review Authority (Dnr: 2019-03986). Participants provided written informed consent.

\section{Consent for publication}

Not applicable.

\section{Competing interests}

The authors declare that they have no competing interests.

\section{Author details}

'Department of Surgical Sciences, Unit of Medical Epidemiology, The EpiHub, Uppsala University, Dag Hammarskjölds väg 14 B, 75185 Uppsala, Sweden. ${ }^{2}$ Department of Medicine, University of North Carolina School of Medicine, Chapel Hill, NC, USA. ${ }^{3}$ Department of Epidemiology, Gillings School of Global Public Health, University of North Carolina, Chapel Hill, NC, USA. ${ }^{4}$ Unit of Cardiovascular and Nutritional Epidemiology, Institute of Environmental Medicine, Karolinska Institutet, Stockholm, Sweden.

Received: 10 December 2020 Accepted: 7 April 2021

Published online: 07 May 2021

\section{References}

1. Banks E, Joshy G, Korda RJ, Stavreski B, Soga K, Egger S, Day C, Clarke NE, Lewington S, Lopez AD. Tobacco smoking and risk of 36 cardiovascular disease subtypes: fatal and non-fatal outcomes in a large prospective Australian study. BMC Med. 2019; 17(1):128. https://doi.org/10.1186/s12916-019-1351-4.

2. Larsson SC, Tektonidis TG, Gigante B, Akesson A, Wolk A. Healthy lifestyle and risk of heart failure: results from 2 prospective cohort studies. Circ Heart Fail. 2016;9(4):e002855. https://doi.org/10.1161/CIRCHEARTFAlLURE.115.002855.

3. Larsson SC, Wolk A, Back M. Alcohol consumption, cigarette smoking and incidence of aortic valve stenosis. J Intern Med. 2017;282(4):332-9. https:// doi.org/10.1111/joim.12630.

4. Larsson SC, Akesson A, Wolk A. Healthy diet and lifestyle and risk of stroke in a prospective cohort of women. Neurology. 2014;83(19):1699-704. https://doi.org/10.1212/WNL.0000000000000954.

5. Larsson SC, Akesson A, Wolk A. Primary prevention of stroke by a healthy lifestyle in a high-risk group. Neurology. 2015;84(22):2224-8. https://doi. org/10.1212/WNL.0000000000001637.

6. Akesson A, Larsson SC, Discacciati A, Wolk A. Low-risk diet and lifestyle habits in the primary prevention of myocardial infarction in men: a population-based prospective cohort study. J Am Coll Cardiol. 2014;64(13): 1299-306. https://doi.org/10.1016/j.jacc.2014.06.1190. 
7. Stackelberg O, Bjorck M, Larsson SC, Orsini N, Wolk A. Sex differences in the association between smoking and abdominal aortic aneurysm. $\mathrm{Br}$ J Surg. 2014;101(10):1230-7. https://doi.org/10.1002/bjs.9526.

8. Larsson SC, Mason AM, Back M, Klarin D, Damrauer SM, Million Veteran P, et al. Genetic predisposition to smoking in relation to 14 cardiovascular diseases. Eur Heart J. 2020;41(35):3304-10. https://doi.org/10.1093/eurheartj/ehaa193.

9. Britton J. Should doctors advocate snus and other nicotine replacements? Yes. BMJ. 2008;336(7640):358. https://doi.org/10.1136/bmj.39479.427477.AD

10. Clarke E, Thompson K, Weaver S, Thompson J, O'Connell G. Snus: a compelling harm reduction alternative to cigarettes. Harm Reduct J. 2019; 16(1):62. https://doi.org/10.1186/s12954-019-0335-1.

11. Rutqvist LE, Curvall M, Hassler T, Ringberger T, Wahlberg I. Swedish snus and the GothiaTek(R) standard. Harm Reduct J. 2011;8(1):11. https://doi. org/10.1186/1477-7517-8-11.

12. Sweden S. Less smoking, more snus using [in Swedish]; 2018.

13. Piano MR, Benowitz NL, Fitzgerald GA, Corbridge S, Heath J, Hahn E, et al. Impact of smokeless tobacco products on cardiovascular disease: implications for policy, prevention, and treatment: a policy statement from the American Heart Association. Circulation. 2010;122(15):1520-44. https:// doi.org/10.1161/CIR.0b013e3181f432c3.

14. Benowitz NL, Hukkanen J, Jacob P 3rd. Nicotine chemistry, metabolism, kinetics and biomarkers. Handb Exp Pharmacol. 2009;192:29-60.

15. Benowitz NL, Burbank AD. Cardiovascular toxicity of nicotine: implications for electronic cigarette use. Trends Cardiovasc Med. 2016;26(6):515-23. https://doi.org/10.1016/j.tcm.2016.03.001.

16. Hergens MP, Lambe M, Pershagen G, Ye W. Risk of hypertension amongst Swedish male snuff users: a prospective study. J Intern Med. 2008;264(2): 187-94. https://doi.org/10.1111/j.1365-2796.2008.01939.x.

17. Haass M, Kubler W. Nicotine and sympathetic neurotransmission. Cardiovasc Drugs Ther. 1997;10(6):657-65. https://doi.org/10.1007/BF00053022.

18. Hansson J, Galanti MR, Hergens MP, Fredlund P, Ahlbom A, Alfredsson L, Bellocco R, Eriksson M, Hallqvist J, Hedblad B, Jansson JH, Nilsson P, Pedersen N, Trolle Lagerros Y, Östergren PO, Magnusson C. Use of snus and acute myocardial infarction: pooled analysis of eight prospective observational studies. Eur J Epidemiol. 2012;27(10):771-9. https://doi.org/1 0.1007/s10654-012-9704-8.

19. Rostron BL, Chang J, Anic GM, Tanwar M, Chang CM, Corey CG. Smokeless tobacco use and circulatory disease risk: a systematic review and meta-analysis. Open Heart. 2018;5(2):e000846. https:/doi.org/10.1136/openhrt-2018-000846.

20. Yatsuya $H$, Folsom AR, Investigators A. Risk of incident cardiovascular disease among users of smokeless tobacco in the Atherosclerosis Risk in Communities (ARIC) study. Am J Epidemiol. 2010;172(5):600-5. https://doi. org/10.1093/aje/kwq191

21. Arefalk G, Hergens MP, Ingelsson E, Arnlov J, Michaelsson $K$, Lind L, et al. Smokeless tobacco (snus) and risk of heart failure: results from two Swedish cohorts. Eur J Prev Cardiol. 2012;19(5):1120-7. https://doi.org/10.1177/1741 826711420003.

22. Henley SJ, Thun MJ, Connell C, Calle EE. Two large prospective studies of mortality among men who use snuff or chewing tobacco (United States). Cancer Causes Control. 2005;16(4):347-58. https://doi.org/10.1007/s10552004-5519-6.

23. Hansson J, Galanti MR, Hergens MP, Fredlund P, Ahlbom A, Alfredsson L, Bellocco R, Engström G, Eriksson M, Hallqvist J, Hedblad B, Jansson JH, Pedersen NL, Trolle Lagerros Y, Östergren PO, Magnusson C. Snus (Swedish smokeless tobacco) use and risk of stroke: pooled analyses of incidence and survival. J Intern Med. 2014;276(1):87-95. https://doi.org/10.1111/joim.12219.

24. Haglund B, Eliasson M, Stenbeck M, Rosen M. Is moist snuff use associated with excess risk of $\mathrm{HD}$ or stroke? A longitudinal follow-up of snuff users in Sweden. Scand J Public Health. 2007;35(6):618-22. https://doi.org/10.1 080/14034940701436949

25. Hergens MP, Galanti R, Hansson J, Fredlund P, Ahlbom A, Alfredsson L, Bellocco R, Eriksson M, Fransson El, Hallqvist J, Jansson JH, Knutsson A, Pedersen N, Lagerros YT, Östergren PO, Magnusson C. Use of Scandinavian moist smokeless tobacco (snus) and the risk of atrial fibrillation. Epidemiology. 2014;25(6):872-6. https://doi.org/10.1097/EDE. 0000000000000169.

26. Larsson SC, Drca N, Jensen-Urstad M, Wolk A. Combined impact of healthy lifestyle factors on risk of atrial fibrillation: prospective study in men and women. Int J Cardiol. 2016;203:46-9. https://doi.org/10.1016/j.jjcard.2015.10.106.

27. Textor J, Hardt J. DAGitty: a graphical tool for analyzing causal diagrams. Epidemiology. 2011;22(5):745. https://doi.org/10.1097/EDE.0b013e318225c2be.
28. Stewart J, Manmathan G, Wilkinson P. Primary prevention of cardiovascular disease: a review of contemporary guidance and literature. JRSM Cardiovasc Dis. 2017;6:2048004016687211.

29. West R. Tobacco smoking: health impact, prevalence, correlates and interventions. Psychol Health. 2017;32(8):1018-36. https://doi.org/10.1080/ 08870446.2017 .1325890

30. Asthana S, Labani S, Kailash U, Sinha DN, Mehrotra R. Association of smokeless tobacco use and oral cancer: a systematic global review and meta-analysis. Nicotine Tob Res. 2019;21(9):1162-71. https:/doi.org/10.1093/ntr/nty074.

31. Vidyasagaran AL, Siddiqi K, Kanaan M. Use of smokeless tobacco and risk of cardiovascular disease: a systematic review and meta-analysis. Eur J Prev Cardiol. 2016;23(18):1970-81. https://doi.org/10.1177/2047487316654026.

32. Sinha DN, Suliankatchi RA, Gupta PC, Thamarangsi T, Agarwal N, Parascandola M, Mehrotra R. Global burden of all-cause and cause-specific mortality due to smokeless tobacco use: systematic review and metaanalysis. Tob Control. 2018;27(1):35-42. https://doi.org/10.1136/toba ccocontrol-2016-053302

33. Hergens MP, Alfredsson L, Bolinder G, Lambe M, Pershagen G, Ye W. Longterm use of Swedish moist snuff and the risk of myocardial infarction amongst men. J Intern Med. 2007;262(3):351-9. https://doi.org/10.1111/j.13 65-2796.2007.01816.x.

34. Janzon E, Hedblad B. Swedish snuff and incidence of cardiovascular disease. A population-based cohort study. BMC Cardiovasc Disord. 2009;9(1):21. https://doi.org/10.1186/1471-2261-9-21.

35. Hergens MP, Lambe M, Pershagen G, Terent A, Ye W. Smokeless tobacco and the risk of stroke. Epidemiology. 2008;19(6):794-9. https://doi.org/10.1 097/EDE.0b013e3181878b33.

36. Hansson J, Pedersen NL, Galanti MR, Andersson T, Ahlbom A, Hallqvist J, Magnusson C. Use of snus and risk for cardiovascular disease: results from the Swedish Twin Registry. J Intern Med. 2009;265(6):717-24. https://doi. org/10.1111/j.1365-2796.2009.02081.x.

37. Folkesson M, Sadowska N, Vikingsson S, Karlsson M, Carlhall CJ, Lanne T, et al. Differences in cardiovascular toxicities associated with cigarette smoking and snuff use revealed using novel zebrafish models. Biol Open. 2016:5(7):970-8. https://doi.org/10.1242/bio.018812.

38. Antoniewicz L, Novo M, Bosson J, Lundback M. Brief exposure to Swedish snus causes divergent vascular responses in healthy male and female volunteers. PLoS One. 2018;13(4):e0195493. https://doi.org/10.1371/journal. pone.0195493.

39. Benowitz NL, Jacob P 3rd, Jones RT, Rosenberg J. Interindividual variability in the metabolism and cardiovascular effects of nicotine in man. Pharmacol Exp Ther. 1982;221(2):368-72.

40. Rohani M, Agewall S. Oral snuff impairs endothelial function in healthy snuff users. J Intern Med. 2004;255(3):379-83. https://doi.org/10.1046/j.1365-2796.2 003.01279.x

41. Westman EC. Does smokeless tobacco cause hypertension? South Med J. 1995;88(7):716-20. https://doi.org/10.1097/00007611-199507000-00004.

42. Oakes JM, Fuchs RM, Gardner JD, Lazartiques E, Yue X. Nicotine and the renin-angiotensin system. Am J Physiol Regul Integr Comp Physiol. 2018; 315(5):R895-906. https://doi.org/10.1152/ajpregu.00099.2018.

43. Adamopoulos D, Argacha JF, Gujic M, Preumont N, Degaute JP, van de Borne P. Acute effects of nicotine on arterial stiffness and wave reflection in healthy young non-smokers. Clin Exp Pharmacol Physiol. 2009;36(8):784-9. https://doi.org/10.1111/j.1440-1681.2009.05141.x.

44. Mehta MC, Jain AC, Mehta A, Billie M. Cardiac arrhythmias following intravenous nicotine: experimental study in dogs. J Cardiovasc Pharmacol Ther. 1997:2(4):291-8. https://doi.org/10.1177/107424849700200407.

45. Wang L, Kittaka M, Sun N, Schreiber SS, Zlokovic BV. Chronic nicotine treatment enhances focal ischemic brain injury and depletes free pool of brain microvascular tissue plasminogen activator in rats. J Cereb Blood Flow Metab. 1997;17(2):136-46. https:/doi.org/10.1097/00004647-199702000-00002.

46. Hawkins BT, Abbruscato TJ, Egleton RD, Brown RC, Huber JD, Campos CR, Davis TP. Nicotine increases in vivo blood-brain barrier permeability and alters cerebral microvascular tight junction protein distribution. Brain Res. 2004;1027(1-2):48-58. https://doi.org/10.1016/j.brainres.2004.08.043.

47. Asplund K. Smokeless tobacco and cardiovascular disease. Prog Cardiovasc Dis. 2003;45(5):383-94. https://doi.org/10.1016/50033-0620(03)80002-6.

\section{Publisher's Note}

Springer Nature remains neutral with regard to jurisdictional claims in published maps and institutional affiliations. 